\title{
Characterization of HicAB toxin-antitoxin module of Sinorhizobium meliloti
}

\author{
Manon Thomet, Annie Trautwetter, Gwennola Ermel ${ }^{*}$ (D) and Carlos Blanco
}

\begin{abstract}
Background: Toxin-antitoxin (TA) systems are little genetic units generally composed of two genes encoding antitoxin and toxin. These systems are known to be involved in many functions that can lead to growth arrest and cell death. Among the different types of TA systems, the type II gathers together systems where the antitoxin directly binds and inhibits the toxin. Among these type II TA systems, the HicAB module is widely distributed in free-living Bacteria and Archaea and the toxin HicA functions via RNA binding and cleavage. The genome of the symbiotic Sinorhizobium meliloti encodes numerous TA systems and only a few of them are functional. Among the predicted TA systems, there is one homologous to HicAB modules.

Results: In this study, we characterize the HicAB toxin-antitoxin module of S. meliloti. The production of the HicA of S. meliloti in Escherichia coli cells abolishes growth and decreases cell viability. We show that expression of the $\mathrm{HicB}$ of S. meliloti counteracts HicA toxicity. The results of double hybrid assays and co-purification experiments allow demonstrating the interaction of HicB with the toxin HicA. Purified HicA, but not HicAB complex, is able to degrade ribosomal RNA in vitro. The analysis of separated domains of HicB protein permits us to define the antitoxin activity and the operator-binding domain.

Conclusions: This study points out the first characterization of the HicAB system of the symbiotic S. meliloti whereas $\mathrm{HicA}$ is a toxin with ribonuclease activity and $\mathrm{HicB}$ has two domains: the $\mathrm{COOH}$-terminal one that binds the operator and the NH2-terminal one that inhibits the toxin.
\end{abstract}

Keywords: Toxin antitoxin, hicAB, BACTH, DNA binding, Sinorhizobium meliloti, RNase

\section{Background}

Toxin antitoxin modules (TA) play important roles in plasmids and prophage stability and are also important actors in bacterial physiology [1]. Canonical TA modules encode a bacterial toxin and a more labile antitoxin in an operon, the antitoxin typically represses the transcription of the operon. The degradation of the antitoxin relieves repression and releases the toxin [2]. The antitoxin could be an RNA molecule (types I and III of TA systems) that controls the level of the toxin protein by either blocking translation of the toxin mRNA or impeding the toxin protein. The nature of the antitoxins may also be proteins that inhibit the toxin by direct interaction or as a hindrance to the effect on the targets (types II and IV of TA systems) [1]. Another types V and

\footnotetext{
* Correspondence: gwennola.ermel@univ-rennes1.fr

Ribosome, bacteria and stress Team, Univ. Rennes, CNRS, Institut de Génétique et de Développement de Rennes (IGDR), UMR6290, F35000 Rennes, France
}

VI were described recently. The antitoxin GhoS, which cleaves the mRNA of the toxin GhoT, is classified in the type V of TA system [3]. The type VI corresponds to the antitoxin that presents the toxin to the proteolytic complex ClpXP [4].

The expressions of TA modules are induced by stress [5]. The links between TA modules and adaptation to stresses are numerous such as the coordination of metabolism with the external supply of nutrients [6]. Whereas the TA systems remain quiescent under favourable growth conditions because of the antagonist action of the antitoxin, under stress, the antitoxins are degraded, allowing the toxins to inhibit essential cellular processes. This inhibition ensues in rapid growth arrest $[7,8]$. The actions of the toxins are diversified: cleavage, modification and degradation of the cellular targets and, finally hamper the bacterial physiology. Numerous toxins are ribosome-dependent or -independent endonucleases. Other actions of the toxins are described such as

(c) The Author(s). 2019 Open Access This article is distributed under the terms of the Creative Commons Attribution 4.0 International License (http://creativecommons.org/licenses/by/4.0/), which permits unrestricted use, distribution, and 
the post-translational modification of the targets or the depolarization of the bacterial membrane leading to the arrest of the ATP synthesis [1]. The toxins could have either indirect effects i.e. MqsR, which is a ribosome independent mRNA interferase, cleaves the ygiS mRNA resulting in increased bile tolerance [9]; or domino effects where one toxin could control another TA module: MqsR cleaves antitoxin ghoS-ghoT mRNA in the ghoS coding region leading to the enhancement of ghoT coding region [10]. The physiological consequence of TA expression could also result of a direct role of the antitoxin on targets other than its cognate toxin: MqsA and DinJ antitoxins are able to repress rpoS gene, affecting the general stress response $[11,12]$. TA modules are involved in the virulence of Salmonella [13], Haemophilus influenza [14] and Staphylococcus aureus [15]. Many of them are implicated in persistence [1] and are considered as targets for antibacterial development. However, the evidence for a role of TA systems in persister cell formation is controversial [16-18].

The HicAB system belonging to the type II TA systems are found in many bacteria and archaea and has been shown to be involved in the stress response virulence and persistence [19-22]. The first identification of hic $A B$ was done in Haemophilus influenza [23] and the term hic came from the localization near the pathogenicity island hif. Then hic $A B$ operons were identified in other bacteria such as Escherichia coli, Burkholderia pseudomallei, Yersinia pestis, Pseudomonas aeruginosa and the structure of HicAB was determined in Yersinia pestis and Streptococcus pneumoniae $[19,24-26]$. In E. coli $\mathrm{K} 12$, the hicAB locus encodes the toxin HicA that cleaves mRNAs and also the tmRNA by a ribosome-independent manner, and the antitoxin HicB [27].

In Sinorhizobium meliloti 53 TA modules were predicted [28], only a few of them were characterized. $\mathrm{NtpP} / \mathrm{R}$ and $\mathrm{VapB} / \mathrm{C}$ are involved in symbiotic efficiency $[29,30]$. Systematic deletion of TA loci in megaplasmids pSymA and pSymB shows that the deletion of some of them affects growth [31], suggesting that part of them is not functional. In this study, we focused on the TA module HicAB of $S$. meliloti corresponding to SMc04441 and SMc04269 hypothetical proteins as defined by Capela et al., [32]. The HicAB modules are highly subjected to horizontal gene transfer and are widely distributed in free-living Bacteria- and Archaea-species, and are not found in genomes of obligate parasites and symbionts [26] such as S. meliloti. Moreover, this HicAB module is involved in virulence and adaptative traits: attenuated virulence of $Y$. pestis mutants [33] development of persister cells in the bacteria B. pseudomaellei [19]; adaptation to extracellular stresses in E. coli [21]. In this study, we show that the operon consisting of the ORFs SMc04441 and SMc04269 in S. meliloti encodes a TA module composed of the functional toxin HicA and antitoxin HicB.

\section{Results \\ hic $A B$ of $S$. meliloti encodes a functional TA system}

In order to study the HicAB module of $S$. meliloti, we assumed that several TA systems could be active in heterologous bacteria [34]. Considering this hypothesis that the TA systems possess a relatively broad host range, we examined the functionality of the HicAB module of $S$. meliloti by constructing a conditional system for the expressions of HicA and HicB in Escherichia coli.

The hicA ORF of S. meliloti, cloned in the pBB-HicA allows the production of HicA under control of the $\mathrm{P}_{\mathrm{BAD}}$ promoter. After induction with arabinose, the influence of the expression of HicA on cell growth was investigated in E. coli strain MG1655 (Fig. 1). Cells carrying the empty pBBara expression vector showed no difference in growth or in viability when they were either induced or not by arabinose. When cells harbouring pBB-HicA were grown without arabinose, they exhibited the same growth pattern estimated by turbidity $\left(\mathrm{OD}_{570 \mathrm{~nm}}\right)$ but when HicA production was induced, growth was abolished (Fig. 1a). Moreover, the number of CFU dramatically reduced after induction and continuously decreased throughout time representative of the lethal effect of the toxin (Fig. 1b).

To investigate whether HicB could alleviate HicA toxicity, hicB ORF was cloned in the plasmid pNDM220. This vector is compatible with pBBara and the expression of HicB is inducible with IPTG. Plasmid derivatives from both vectors were introduced in trans and induced simultaneously in E. coli. After two hours of induction, cells carrying the empty vector pNDM220 and pBBHicA exhibited lethality (Fig. 1c, line B) whereas in cells harbouring pBB-HicA and pNDM-HicB for which the simultaneous production of HicA and HicB reduced drastically HicA lethal effect (Fig. 1c, line D).

\section{HicB binds to hic $A B$ promoter region}

In type II TA systems the transcription of both toxin and antitoxin are autoregulated: the antitoxin binds to the promoter of the TA operon and thus inhibits transcription of both toxin and antitoxin [1]. To study HicB interaction with hic $A B$ promoter, we performed an electrophoretic mobility shift assay (EMSA) using purified His-tagged $\mathrm{HicB}$ and a $223 \mathrm{bp}$ sequence containing the promoter of genes hic $A B$, which was previously characterized [35, 36] (Fig. 2). An upshifted band appeared faintly with a low concentration $(50 \mathrm{nM})$ of $\mathrm{HicB}$ and was clearly present with the highest concentrations (from 0,25 to $1 \mu \mathrm{M}$ ) of HicB. These results demonstrate that the HicB protein of $S$. meliloti binds to the hicAB promoter region. 


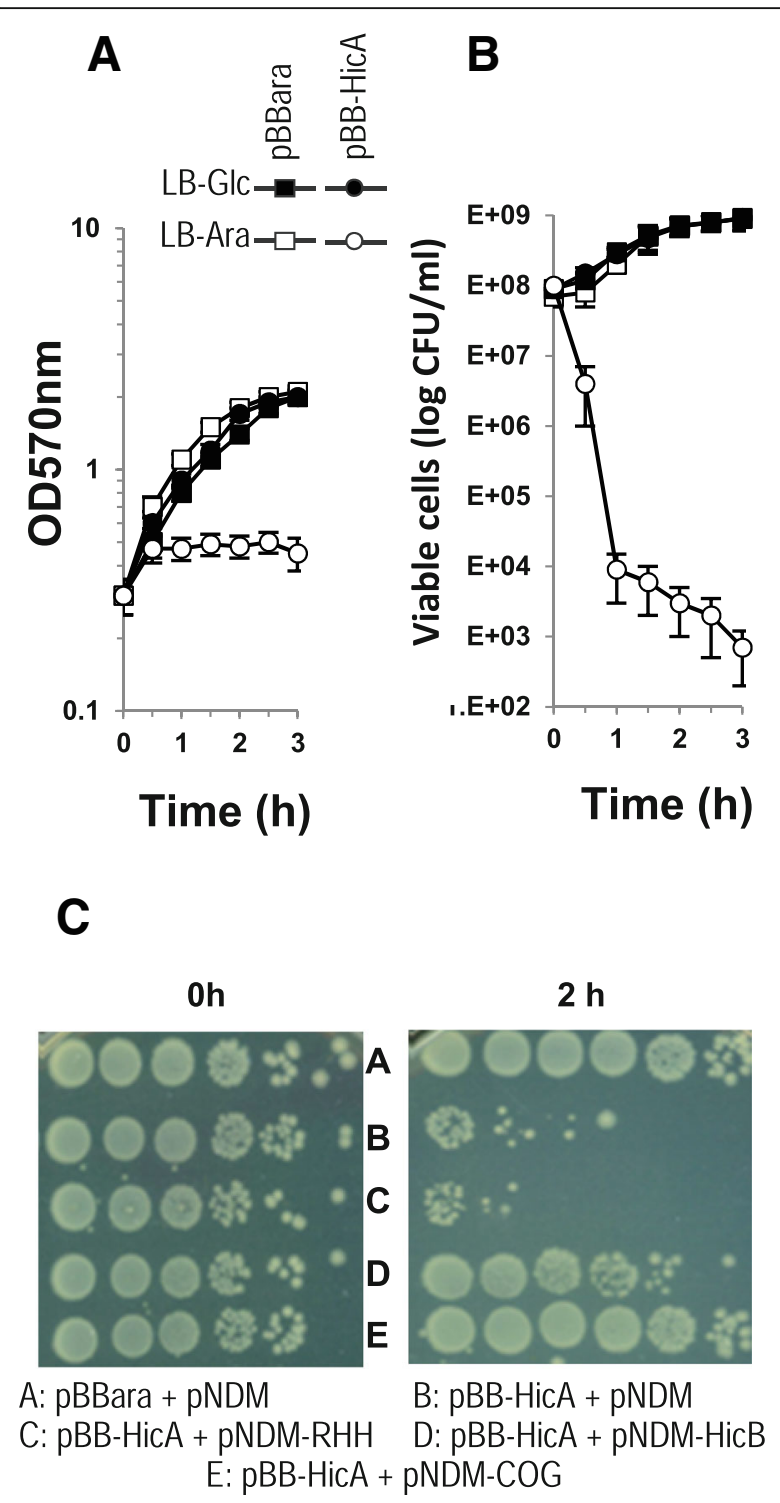

Fig. $1 \mathrm{HicB}$ prevents the toxicity of HicA in E. coli. E. coli strain MG1655 containing pBBara (squares) or pBB-HicA (circles) were precultured in $\mathrm{LB}$ medium containing $10 \mathrm{mM}$ glucose to an $\mathrm{OD}_{570 \mathrm{~nm}}$ of 0.2 . After centrifugation, cells were suspended in LB medium containing $10 \mathrm{mM}$ glucose (black symbols) or $10 \mathrm{mM}$ arabinose (white symbols). The growth of the strains was then followed for $3 \mathrm{~h}$ and estimated using either turbidity $\left(\mathrm{OD}_{570 \mathrm{~nm}}\right.$, a) or viable cell counts (log (CFU/mL), b). The panel $\mathbf{c}$ corresponds to the numeration of the E. coli MG1655 cells containing pBBara + pNDM (A), pBB-HicA + pNDM (B), pBB-HicA + pNDM-RHH (C), pBB-HicA + pNDM-HicB (D), pBB-HicA + pNDM-COG (E) after $2 \mathrm{~h}$ of arabinose induction in $\mathrm{LB}$ medium, the details of the procedure were as in A. Tenfold dilutions were spotted on a LB plate and incubated for $48 \mathrm{~h}$

\section{HicB interacts with HicA}

To assess HicA-HicB protein interactions, we used the bacterial two-hybrid system (BACTH) in E. coli cya strain BTH101. BACTH relies on the recovery of Bordetella pertussis adenylate cyclase whose domains T18 and T25 are

\section{HicB $(\mu \mathrm{M})$

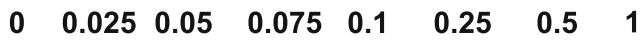

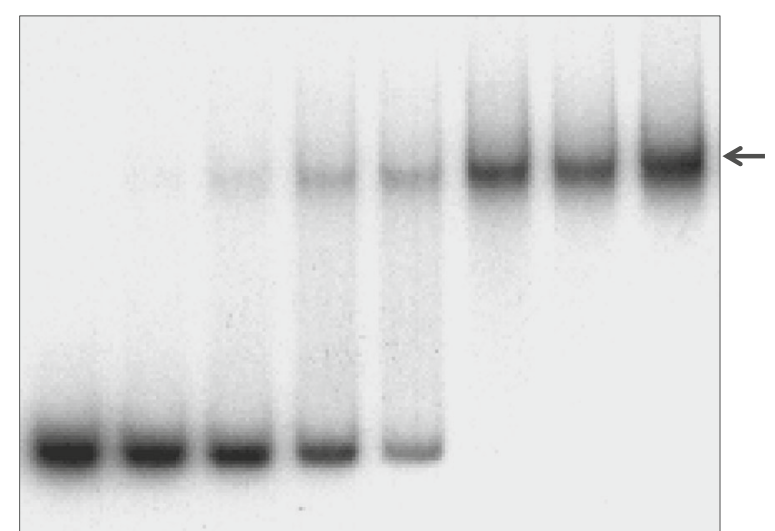

Fig. 2 HicB binds hicAB promoter region. Gel mobility shift analysis of specific binding of the purified $\mathrm{HicB}$ to the promoter region of hicAB operon. The probe, a 223 bp DNA fragment amplified by PCR, was incubated with increasing amounts of purified HicB (0 to $1 \mu \mathrm{M})$ and submitted to electrophoresis in a 5\% PAGE in Tris acetate buffer $\mathrm{pH}$ 7.5. The position of the protein-DNA complex is indicated by an arrow

split and can be fused with proteins [37]. HicA and HicB were alternatively fused to the $\mathrm{NH} 2$ and $\mathrm{COOH}$ ends of T18 and T25 domains in different combinations, for which $\beta$-galactosidase assays were monitored (Additional file 1: Figure S1). All the combinations between $\mathrm{HicA}$ and HicB showed significant $\beta$-galactosidase activities in regards to those determined with empty vectors without any fused protein and self-interactions of HicA. High $\beta$-galactosidase activity was detected in cells producing T25-HicA and T18-HicB reflecting HicA-HicB interaction (Fig. 3a). Although HicA does not self-interact the co-expression of T25-HicB and T18-HicB resulted in high levels of $\beta$-galactosidase activity (Fig. 3a) suggesting HicB self-interaction and a possible dimeric organization of the protein.

To confirm HicA-HicB interaction, we performed co-purification experiments. A synthetic hicAstrep-hicBhis operon (Additional file 2: Figure S2) was introduced in $\mathrm{pET} 22 \mathrm{~b}(+)$ vector leading to the productions of strep-tagged HicA and his-tagged $\mathrm{HicB}$ at their $\mathrm{COOH}$ ends. After IPTG induction, the produced proteins were alternatively purified on IMAC chromatography and on Strep-Tactin resins. In both cases HicA and HicB were co-purified (Fig. 3b).

These data indicate that S. meliloti HicA and HicB interact, as expected for a toxin-antitoxin system. Moreover HicB would be a multimeric protein while HicA behaves as a monomer as this has been shown using the two-hybrid assays and described for HicAB in $Y$. pestis [24]. 


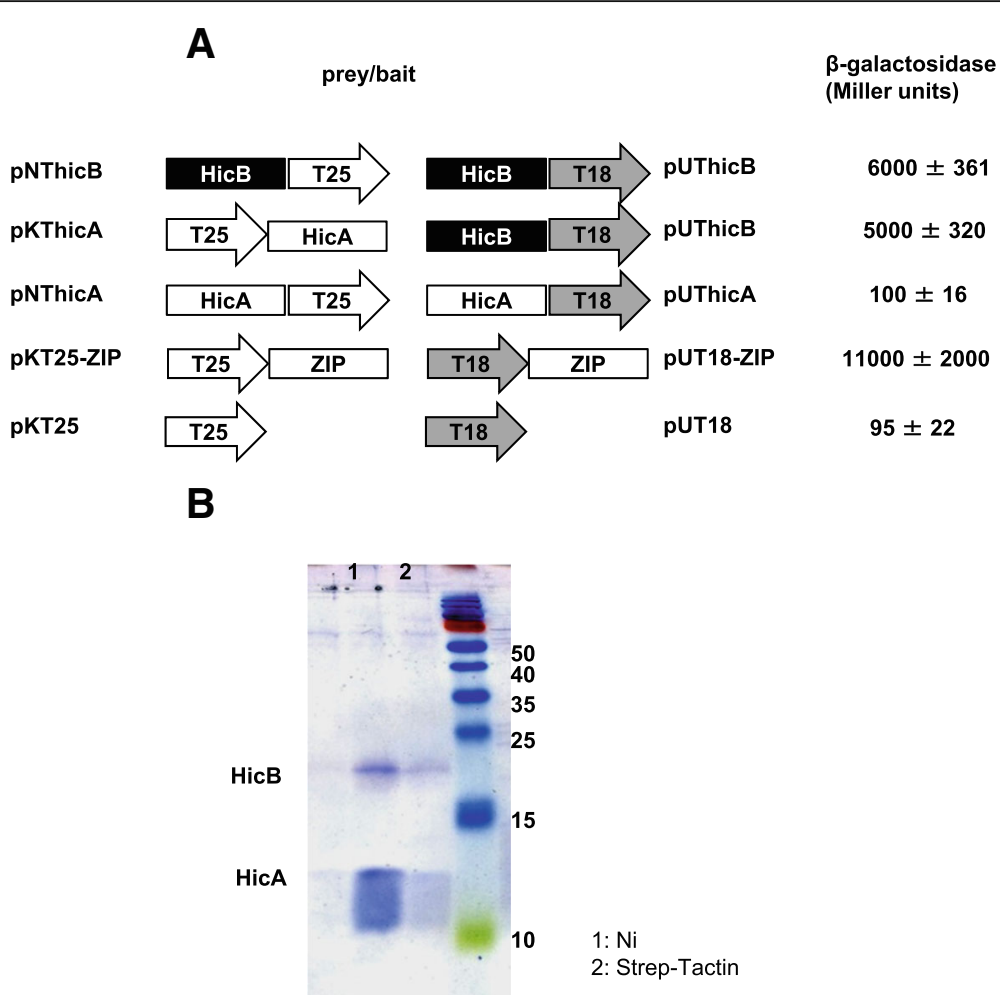

Fig. 3. HicB interacts with HicA. a Two hybrid analysis of HicA and HicB interaction. HicB (black squares) and HicA (white squares) were fused to T25 (white arrow) or T18 (grey arrow) catalytic domains of Bordetella pertussis adenylate cyclase both at the $\mathrm{N}$ or $\mathrm{C}$ terminus of these domains. The whole set of combinations is presented in Fig.S1. For this purpose hicA and hicB open reading frames were amplified by PCR using ToxXbaToxEco and DopXba-DopEco primers (Table 3). The amplicons cleaved by Xbal and EcoRI were cloned into pKT25, pKNT25, pUT18 and pUT18C, the resulting plasmids are listed in Table 2. After introduction of the different recombinant plasmids into E. coli strain BTH101, $\beta$-galactosidase activities were assayed after growth in LB medium containing $1 \mathrm{mM}$ IPTG. Empty vectors pUT18 and pKNT25 were used to determine basal level of $\beta$-galactosidase activity and pKT25-Zip and pUT18-Zip as positive control of a high interaction. $\beta$-galactosidase activity (Miller units) is indicated, results are the average of three independent experiments. b. SDS-PAGE of both HicA-Strep and HicB-6His. A synthetic operon hicA-strep, hicB-6His was cloned in pET22b(+). E. coli BL21 containing this plasmid was grown in LB medium to an $\mathrm{OD}_{570 \mathrm{~nm}}$ of 0.6, $1 \mathrm{mM}$ IPTG was added, and the growth of cells was carried on three hours after induction. Cells were collected and broken, protein extract was applied to Ni affinity chromatography using imidazole for elution (lane 1) and Strep-Tactin affinity chromatography using desthiobiotin for elution (lane 2). The eluted proteins were resolved on a $12.5 \%$ SDS PAGE.

\section{Antitoxin stability and toxin purification}

Toxins are stable proteins while antitoxins are targeted by proteases such as the Lon protease or by ClpP associated with either ClpA or ClpX [2]. Proteases synthesis is induced by stresses such as osmotic and starvation ones. Thus we hypothesized that the strains expressing toxin and antitoxin must have their growth affected by stress while it would not be affected in non-stressing medium. The E. coli strain MG1655 containing both pBB-HicA and pNDM-HicB, or both pBB-HicA and pNDM220, or both pBBara and pNDM220 vectors as controls, was grown in M63 medium after induction of hicA and hicB. The sole induction of HicA and HicB production did not affect growth (data not shown). A growth arrest was observed when osmotic stress (addition of $\mathrm{NaCl}$ to media) was applied to strains with induced expressions of toxin and antitoxin (Fig. 4 a). The strains containing empty vectors still grew (data not shown). These results showed that the cell survival was affected after stress application. Concerning the strain containing both pBB-HicA and pNDM220, defect of growth was observed after arabinose induction leading to the production of the toxin HicA. A concentration of $0.5 \mathrm{M} \mathrm{NaCl}$ in M63 medium amplified this defect of growth.

The extraction of proteins was performed in E. coli BL21 cells harbouring pETHicAStrepHicBHis $3 \mathrm{~h}$ after IPTG induction, and $4 \mathrm{~h}$ after $\mathrm{NaCl}$ addition (i.e. $7 \mathrm{~h}$ after induction); proteins were loaded on Strep-Tactin column that traps the strep-tagged toxin. Toxin and antitoxin were eluted from extracts of non-salted cultures (Fig. 4 b) while only toxin was eluted from salted cultures (Fig. 4 c). These data show that the antitoxin produced before $\mathrm{NaCl}$ addition is degraded during salt stress adaptation.

To determine the toxic effect of HicA, RNase activity of purified proteins was analysed. Extracts of cells 
A

pBBara + pNDM220

pBB-HicA + pNDM-HicB

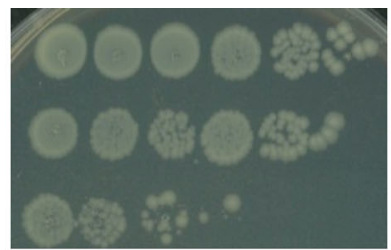

OM NaCl

pBB-HicA + pNDM220

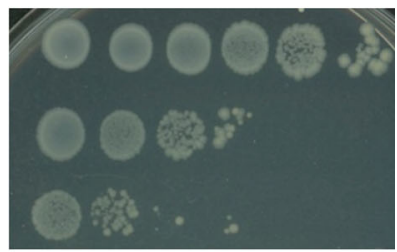

$0.5 \mathrm{M} \mathrm{NaCl}$

pBB-HicA + pNDM220

B

C

D

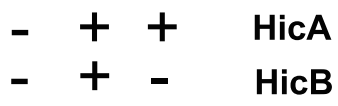
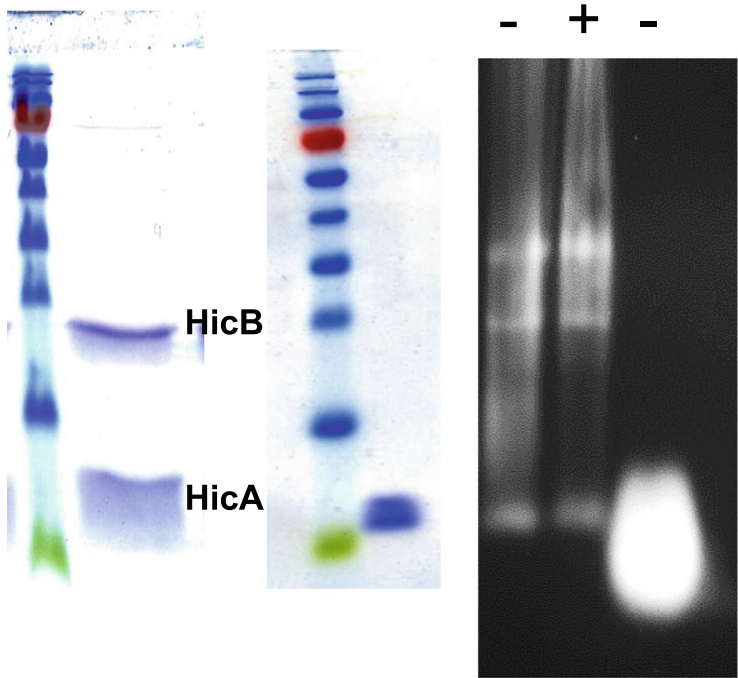

Fig. $4 \mathrm{HicB}$ stability and RNase assay. Viable cell counts of cells of E. coli strain MG1655 containing pBBara and pNDM220, pBB-hicA and pNDMhicB or pBB-hicA and pNDM220 that were grown in M63 medium containing or not $0.5 \mathrm{M} \mathrm{NaCl}$ (a). Strains were induced with $1 \mathrm{mM}$ arabinose and $1 \mathrm{mM} \mathrm{IPTG}$. Viable cell counts (ten fold dilutions) were determined throughout time, the figure illustrates results obtained $3 \mathrm{~h}$ after induction. Cells of E. coli strain BL21 containing pETHicAStrep-HicBHis grown in M63 medium were induced with $1 \mathrm{mM}$ IPTG for 3 h. Half of the culture was centrifuged and protein content was loaded on Strep Tactin and eluted with $1 \mathrm{mM}$ desthiobiotin (b). The other half of the culture was added with $0.5 \mathrm{M} \mathrm{NaCl}$ and incubation was extended for $4 \mathrm{~h}$, then the protein content was loaded on Strep-Tactin and eluted with desthiobiotin (c). $20 \mathrm{\mu g}$ of eluted proteins were solved by SDS PAGE (b, c). The same amount of eluted proteins was incubated with ribosomal RNAs $1 \mathrm{~h}$ at $37^{\circ} \mathrm{C}$ and then loaded on $1 \%$ bleach agarose gel (d).

cultured in M63 medium that contained the HicA-HicB complex did not show RNase activity against purified ribosomal RNAs. In contrast ribosomal RNAs were fully degraded by purified HicA originated from cells cultured in $\mathrm{M} 63$ medium containing $0.5 \mathrm{M} \mathrm{NaCl}$ after $1 \mathrm{~h}$ at $37^{\circ} \mathrm{C}$ (Fig. 4 d).

These results show that contrary to HicB, HicA ribonuclease is not degraded by proteases induced by osmotic stress $[1,2]$. This allows a simple toxin purification protocol that did not need protein denaturation and renaturation as described for other HicA proteins.

\section{HicB is composed of two functional modules}

The structure of HicAB was determined in $Y$. pestis and S. pneumoniae. In both bacteria, HicA and HicB structures are very close $[24,25]$. In these bacteria, HicA is a 
monomer while HicB is dimeric or tetrameric. This antitoxin is composed of two domains linked by a hinge, a $\mathrm{NH} 2$ domain interacting with HicA toxin and a $\mathrm{COOH}$ domain possessing a ribbon-helix-helix $(\mathrm{RHH})$ motif that interacts with the operator. HicB3 of $Y$. pestis dimerizes through the RHH domain while HicB of S. pneumoniae dimerizes through the $\mathrm{NH} 2$ domain.

Clustal alignments of S. meliloti HicA with HicA of $E$. coli, Y. pestis and S. pneumoniae, show that all the proteins are very similar (Additional file 3: Figure S3), the histidine conserved residue important for the activity is conserved in S. meliloti HicA. In contrast, Clustal alignments of HicB show that the amino acids are less conserved in HicB proteins (Fig. S3), the DNA binding domain is not conserved, and only 6 aminoacids are identical in the four proteins, nevertheless no particular function was attributed to them in structural studies [24, 25]. Structure prediction of $S$. meliloti with Phyre server shows that its structure is close to that of $Y$. pestis and $S$. pneumoniae, main differences were observed in the hinge region (Additional file 4: Figure S4 and Additional file 5: Figure S5).

The two domains (RHH for C-terminal and COG for $\mathrm{N}$-terminal domains) were cloned in pBBara, the hinge region was conserved in both constructs. The arabinose induction of the two domains does not induce toxicity in E. coli cells (data not shown). Thus, the sequences corresponding to the two domains were separately cloned into pNDM vector and introduced in $E$. coli with pBB-HicA. After induction with arabinose and IPTG, toxicity was observed when HicA and HicB-RHH were produced simultaneously (Fig. $1 \mathrm{c}$ lane $\mathrm{C}$ ). In contrast expression of HicB-COG domain abolishes HicA toxicity (Fig. 1c lane E). The antitoxin effect is greater than that observed with HicB (Fig. 1c lane E). Interaction of the HicB NH2-domain with HicA was confirmed by BACTH analysis (Fig. 5 and Additional file 6: Figure S6). The HicB COG domain must multimerize as deduced from the high $B$-galactosidase activity observed in HicB-COG self-interaction in BACTH assays (Fig. 5 and

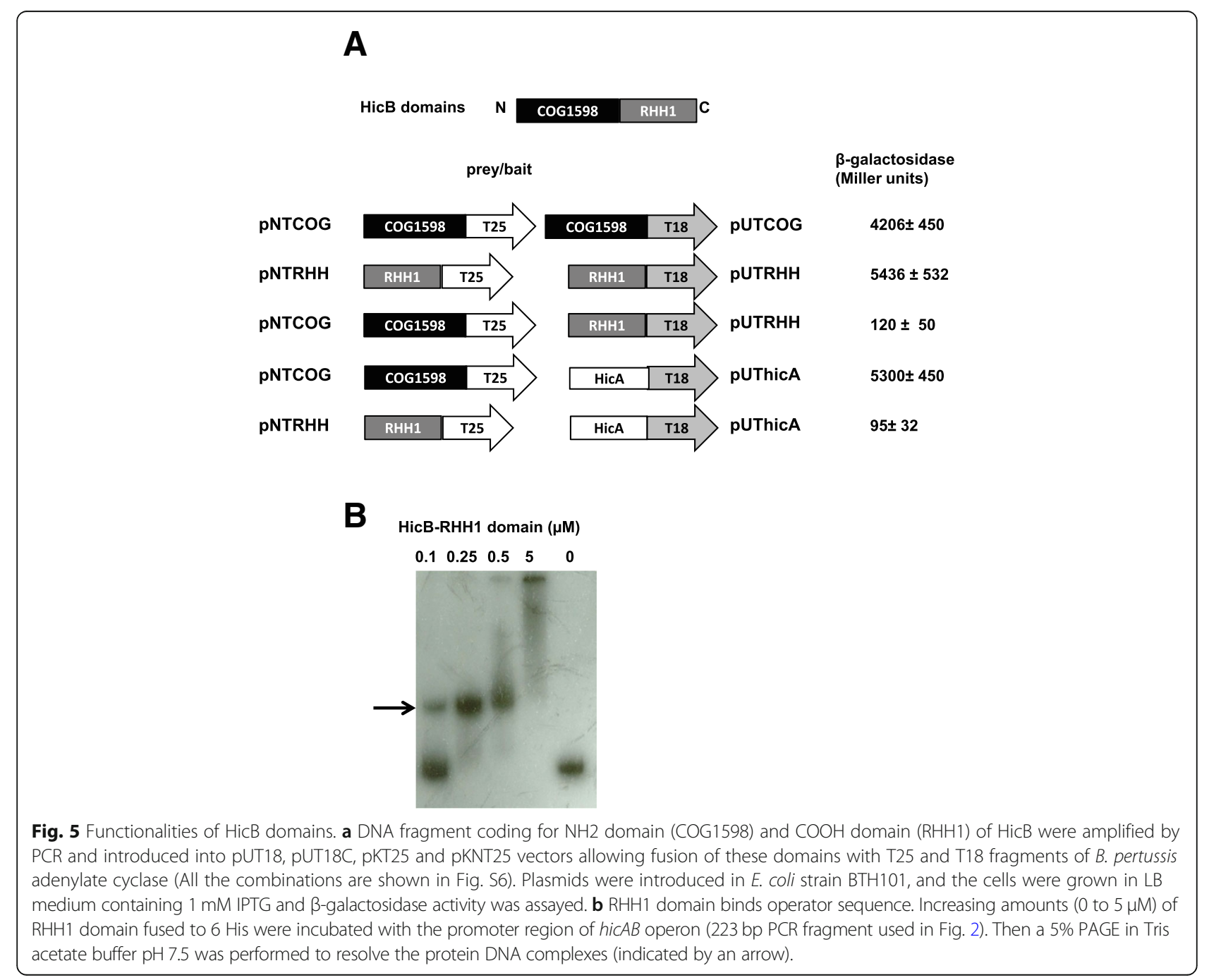


Additional file 5: Figure S5). Moreover, this HicB-COG was unable to interact with HicB-RHH domain in BACTH assays. The HicB RHH domain was purified and used in EMSA studies with HicB binding region. It bound operator sequence as efficiently as HicB protein (Fig. 5). The analysis of BACTH assays showed that $\mathrm{RHH}$ domain dimerizes, a feature necessary for efficient binding to the operator.

\section{Discussion}

Numerous TA modules exist in Bacteria, they are related to numerous functions that allow the control of growth arrest when cells adapt to variable environments. In the nitrogen-fixing soil bacterium $S$. meliloti, numerous TA modules were predicted $[26,28]$ and one TA module, encoded by smc04441/smc04269, showed homologies with hic $A B$ loci [32]. In this study, we demonstrate that the HicAB module of $S$. meliloti is functional in E. coli. The expression of HicA toxin under the control of the arabinose promoter in E. coli permits us to show that HicA toxin provoked a decrease of the viability of bacteria prevented by the simultaneous expression of HicB antitoxin (Fig. 1c). The interactions between the two proteins were confirmed by the two hybrid assays using the BACTH system [37] whatever the combination using HicA or HicB (Fig. 3a and Additional file 1: Figure S1). The co-purification of strep-tagged HicA and His-tagged HicB using affinity chromatographies on either Nickel or Strep-Tactin resins is another argument in favour of HicA-HicB interaction (Fig. 3b). Furthermore, these experiments allow us to reveal a self-interaction and a possible dimerization of $\mathrm{HicB}$ (Additional file 1: Figure S1). These data indicate that $S$. meliloti HicA and HicB interact, as expected for a toxin-antitoxin system. Moreover there are evidence that $\mathrm{HicB}$ would be a multimeric protein while HicA behaves as a monomer, as described for HicAB in Y. pestis [24]. The purified His-tagged HicB bound to the promoter of genes hicAB (Fig. 2a). Thus the HicB protein of S. meliloti binds to the hic $A B$ promoter region such as the antitoxins HicB of other bacteria [24,38].

The simultaneous induction of HicA and HicB affected growth only when osmotic stress was applied by the addition of $\mathrm{NaCl}$ to the medium (Fig. 4a), meaning that the HicB antitoxin was digested by osmotic stress-induced proteases such as Lon or ClpP as this phenomenon has been observed for other antitoxins $[1$, 2]. This particularity was used to purify the HicA toxin only (Fig. 4c) using a simple protocol. The HicA protein of $S$. meliloti was shown to be a ribonuclease that degraded purified ribosomal RNAs (Fig. 4d). RNA cleavage would block translation and cause primarily a growth arrest and finally a decrease of cell viability [27].
The analysis of the predicted structure of HicB showed a NH2 domain with homologies to the domain of HicB of S. pneumoniae and $Y$. pestis, interacting with HicA toxin and a $\mathrm{COOH}$ domain possessing a potential ribbon-helix-helix motif, binding promoter that is less conserved (Additional file 2: Figure S2). The different experiments permitted to show that the two domains are active independently: the $\mathrm{NH} 2$ domain of HicB interacts with the toxin HicA and the $\mathrm{COOH}$ domain of HicB binds to the promoter of hicAB operon. Moreover, these two domains potentially showed self-interaction and a possible dimerization. HicB of $Y$. pestis and $S$ pneumoniae possess only one dimerization domain, but not in the same region. Isolated domains of S. meliloti have both the hinge region, nevertheless we could exclude that it is responsible for dimerization since no interaction was observed between $\mathrm{NH} 2$ and $\mathrm{COOH}$ domains. Thus $S$. meliloti HicB must have a slightly different structure with two interaction regions between monomers. Alternatively, HicB of the other bacteria also has the same ability to dimerize through two regions, but only one is used depending of experimental conditions that were different for $Y$. pestis and S. pneumoniae $[24,25]$.

\section{Conclusion}

In this study we characterize the biochemical properties of HicA and HicB proteins of S. meliloti. Purified HicA has an RNAse activity; HicB is a DNA binding protein. Both proteins interact and this interaction via the $\mathrm{NH} 2$ domain of HicB abolishes the RNAse activity of the toxin. The following work, which is in progress, would have to determine the physiological importance of HicAB TA module in S. meliloti.

\section{Methods}

\section{Bacterial strains and culture conditions}

E. coli and S. meliloti strains are listed in Table 1 . They were grown in LB medium at $37^{\circ} \mathrm{C}$. For the selection of E. coli transformants, antibiotics (Sigma-Aldrich) were added: ampicillin $(50 \mu \mathrm{g} / \mathrm{mL})$, kanamycin $(50 \mu \mathrm{g} / \mathrm{mL})$, neomycin $(50 \mu \mathrm{g} / \mathrm{mL})$, chloramphenicol $(25 \mu \mathrm{g} / \mathrm{mL})$ and tetracycline $(10 \mu \mathrm{g} / \mathrm{mL})$. For the culture of $S$. meliloti strain $102 \mathrm{~F} 34$, streptomycin was used at $100 \mu \mathrm{g} / \mathrm{ml}$.

\section{DNA manipulations}

Chromosomal and plasmid DNA isolations were undertaken according to the standard procedures [39]. Sequencing was performed by Eurofins.

\section{Plasmids, synthetic genes and primers}

The plasmids and oligonucleotides that were used in this study are listed in Tables 2 and 3. The synthetic genes are described in Additional file 2: Figure S2. 
Table 1 Bacterial strains

\begin{tabular}{|c|c|c|}
\hline Strains & Genotypes & References \\
\hline \multicolumn{3}{|l|}{ S. meliloti } \\
\hline $102 F 34$ & WT SmR & [45] \\
\hline \multicolumn{3}{|l|}{ E. coli } \\
\hline MG1655 & $\mathrm{F}^{-}$, lambda ${ }^{-}, \mathrm{rph}-1$ & [46] \\
\hline BL21 & E. coli B F ${ }^{-} d c m$ ompT hsdS ga凡 (DE3) & [47] \\
\hline BTH101 & $F^{\prime}$, cya-99, araD139, galE15, galK16, rpsL1 $\left(S_{t} r^{R}\right)$, hsdR2, mcrA1, mcrB1, relA1 & [48] \\
\hline
\end{tabular}

A new vector, pBBara was constructed: the NsiI-HindIII fragment carrying araC, araO was liberated from pBAD24 and introduced between the corresponding sites of pBBR1-MCS2, which was reported to have five to ten copies per chromosome/cell in E. coli [40].

In order to express HicA, the hicA sequence was amplified from genomic DNA of $S$ meliloti strain 102F34 using the primers toxpBAD-A (carrying a NheI site) and toxpBAD-B (carrying a SalI site). After restriction, the PCR fragment was introduced between the corresponding sites of pBBara, giving the pBB-HicA plasmid.

For the expression of HicA-StrepTag and HicB-6HisTag, a synthetic hic $A B$ operon was synthesized (Eurofins Genomics) (Additional file 2: Fig. S2) with a NdeI restriction site at the initiation codon of hicA, a Strep-Tag (WSHPQFE) at the COOH extremity of hicA, a XhoI site at stop codon of $h i c B$ in order to allow the expression of a 6 His-Tag after the insertion of the fragment into the pET22b(+) vector between the NdeI and XhoI sites. This construct was named pETHicAStrepHicBHis.

The plasmid pET-HicB contains the HicB ORF, which was amplified by PCR from $S$. meliloti genomic DNA using the primers (HicBNde, HicBXho) and inserted into the $\mathrm{pET} 22 \mathrm{~b}(+)$ vector between the restriction sites $\mathrm{NdeI}$ and Xhol, introducing a 6His-Tag at the $\mathrm{COOH}$ extremity of HicB.

The COG1598 (NH2-terminal extremity) and the RHH1 (COOH-terminal extremity) domains of HicB were obtained using amplification with HicBNH2Nde/ HicBNH2Xho and RHHNde/HicBXho respectively and introduced into $\mathrm{pET} 22 \mathrm{~b}(+)$ vector yielding $\mathrm{pET}-\mathrm{COG}$ and pET-RHH respectively.

Primers PET-BglII and PET-EcoRI were used to amplify the sequence of interest from pET-HicB, pET-RHH and pET-COG1598. The amplicons were digested with BglII and EcoRI and cloned between the EcoRI and BamHI sites of pNDM220, yielding pNDM-HicB, pNDM-COG and pNDM-RHH respectively.

\section{Purification of HicA and HicB}

The $\mathrm{pET} 22 \mathrm{~b}(+)$ expression vector possesses a T7 promoter controlled by LacI. All its derivatives were transformed in BL21 cells. pET-HicB, pET-RHH and pET-COG1598 transformants were grown in $\mathrm{LB}$ medium to an $\mathrm{OD}_{570 \mathrm{~nm}}$ of 0.6 ,
IPTG was added at $1 \mathrm{mM}$ and incubation was continued for $4 \mathrm{~h}$. Cells were collected by centrifugation $(5000 \mathrm{~g}, 15$ min), washed (Tris- $\mathrm{HCl} 20 \mathrm{mM}, \mathrm{NaCl} 500 \mathrm{mM}$, imidazole $5 \mathrm{mM}, \mathrm{pH} 8$ ), resuspended in the same buffer, broken in a French press $\left(8,610^{6} \mathrm{~N} \mathrm{~m}^{-2}\right)$ and centrifuged $(20,000 \mathrm{~g}, 30$ min). Protein extracts were loaded on a Ni-NTA agarose resin (Qiagen), washed with $60 \mathrm{mM}$ imidazole buffer and proteins eluted with $200 \mathrm{mM}$ imidazole buffer. Proteins were dialysed against PBS buffer.

BL21 containing pETHicAStrepHicBHis was grown in LB medium to an $\mathrm{OD}_{570 \mathrm{~nm}}$ of 0.6 before $1 \mathrm{mM}$ IPTG was added for protein induction. For purification of HicA-HicB complex, the bacterial cells were harvested 3 $\mathrm{h}$ later. For HicA purification $1 \mathrm{M} \mathrm{NaCl}$ was added $3 \mathrm{~h}$ after induction with IPTG and incubation was continued for $4 \mathrm{~h}$. The purification steps were identical to those described above. Alternatively protein extract was loaded on a Strep-Tactin resin (IBA Lifesciences), and eluted with $2.5 \mathrm{mM}$ desthiobiothin.

\section{RNase assay}

E. coli ribosomes were purified on $10-40 \%$ sucrose gradients. Collected fractions containing $70 \mathrm{~S}$ ribosomes were centrifuged $(150,000 \mathrm{~g}, 2 \mathrm{~h})$ and $70 \mathrm{~S}$ particles re-suspended in $50 \mathrm{mM}$ TRIS pH7.5, $\mathrm{NaCl} 0.75 \mathrm{M}$, MgSO4 10 mM. RNA was extracted with Trizol (Thermo Fisher Scientific) and re-suspended in PBS buffer.

HicA and HicAB complexes were purified on Strep-Tactin resin (IBA Lifesciences) and resuspended in PBS buffer. Ribosomal RNA (130 pM) and proteins (20 $\mathrm{nM}$ ) were incubated at $37^{\circ} \mathrm{C}$ for $1 \mathrm{~h}$ and RNA was analysed on bleach agarose gels [41].

\section{EMSA}

A 221 bp DNA fragment embedding hicAB promoter (213. + 8) was amplified and labelled using PCR with EMSAD and EMSAR primers in the presence of $\left[\alpha^{32]} \mathrm{P}\right.$ $\mathrm{dCTP}$. The binding reaction was performed in a $20 \mu \mathrm{l}$ reaction volume containing10 mM TRIS- $\mathrm{HCl} \mathrm{pH} 7.5,50$ $\mathrm{mM} \mathrm{NaCl}, 1 \mathrm{mM}$ DTT, $300 \mu \mathrm{g} \mathrm{ml}^{-1}$ BSA the labelled DNA fragment and the purified HicB-His6 or RHH-6His.

The reaction was incubated $30 \mathrm{~min}$ at $25^{\circ} \mathrm{C}$ followed by the addition of $2 \mu \mathrm{l}$ of $50 \%$ glycerol and electrophoresis through a $5 \%$ native polyacrylamide gel at $170 \mathrm{~V} \mathrm{~cm}^{-1}$ in 
Table 2 Plasmids used in this study

\begin{tabular}{|c|c|c|}
\hline Names & Genotypes & References \\
\hline pBAD24 & ori ColE1 araC, $\mathrm{P}_{\mathrm{BAD}}$ bla & [49] \\
\hline pNDM220 & Mini R1, bla, $|a c|^{9}, \mathrm{P}_{\mathrm{A} 1 / \mathrm{O} 4 / \mathrm{O} 3}$ & {$[50]$} \\
\hline pBBR1-MCS2 & ori pBBR1, lacZa, aphA & [40] \\
\hline $\mathrm{pET} 22 \mathrm{~b}(+)$ & ori ColE1, bla, lacl, T7p & Novagen \\
\hline pKT25 & ori P15A, aac, T25 & {$[37]$} \\
\hline pKNT25 & ori P15A, aac, T25 & {$[37]$} \\
\hline pUT18 & ori ColE1 bla, T18 & {$[37]$} \\
\hline pUT18C & ori ColE1 bla, T18 & {$[37]$} \\
\hline pKT25-Zip & ori P15A, aac, T25:: Zip & {$[37]$} \\
\hline pUT18-Zip & ori ColE1 bla, T18:: Zip & {$[37]$} \\
\hline pBBara & pBBR1-MCS2, araC, $P_{B A D}$ & \\
\hline pBB-HicA & pBBR1-MCS2, araC, $P_{B A D}-h i c A$ & This study \\
\hline pET-HicB & pET22 hicB 6 his & This study \\
\hline pET-RHH & pET22 RHH1 6 his & This study \\
\hline pET-COG & pET22 COG 6 his & This study \\
\hline pETHicAStrepHicBHis & pET22 synthetic operon & This study \\
\hline pNDM-HicB & pNDM220 hicB & This study \\
\hline pNDM-COG & pNDM220 NH2 fragment of HicB (COG1598) & This study \\
\hline pNDM-RHH & pNDM220 COOH fragment of $\mathrm{HicB}(\mathrm{RHH})$ & This study \\
\hline pKThicA & pKT25 hicA & This study \\
\hline pKThicB & pKT25 hicB & This study \\
\hline pNThicA & pKNT25 hicA & This study \\
\hline pNThicB & pKNT25 hicB & This study \\
\hline pUThicA & pUT18 hicA & This study \\
\hline pUThicB & pUT18 hicB & This study \\
\hline pUChicA & pUT18C hicA & This study \\
\hline pUChicB & pUT18C hicB & This study \\
\hline pKTCOG & pKT25 COG1598 & This study \\
\hline pNTCOG & pKNT25 COG1598 & This study \\
\hline pKTRHH & pKT25 RHH1 & This study \\
\hline pNTRHH & pKNT25 RHH1 & This study \\
\hline pUTCOG & pUT18 COG1598 & This study \\
\hline pUCCOG & pUT18C COG1598 & This study \\
\hline pUTRHH & pUT18 RHH1 & This study \\
\hline pUCRHH & pUT18C RHH1 & This study \\
\hline
\end{tabular}

TAE buffer (20 mM Tris, $40 \mathrm{mM}$ acetate, $1 \mathrm{mM}$ EDTA, $\mathrm{pH}$ 7.5). The gel was then dried and revealed by autoradiography.

\section{BACTH analysis}

HicA and HicB ORFs were amplified by PCR using ToxXbaT/ToxEcoT and DopXba/DopEcoT primer pairs for HicA and HicB respectively and cloned into pKT25, pKNT25, pUT18 and pUT18C vectors between $X b a \mathrm{I}$ and EcoRI restriction sites. Strain BTH101 was transformed with a combination of one pUT (18 or $18 \mathrm{C}$ ) derivative and a $\mathrm{pKT}$ (or $\mathrm{pKNT}$ ) derivative in order to obtain all the combinations to analyse HicA-HicA, HicB-HicB and HicA-HicB interactions. pKT25-ZIP and pUT18-ZIP were used as reference for positive interaction and pKT25 and pUT18 as control for the absence of interaction. BTH101 transformants were grown in LB or M63 medium containing $10 \mathrm{mM}$ glycerol as growth substrate. $\beta$-galactosidase enzymatic activity was assayed after induction with $1 \mathrm{mM}$ IPTG according to [42]. 
Table 3 Oligonucleotides used in this study

\begin{tabular}{|c|c|c|}
\hline Names & Sequences* & Restriction sites \\
\hline Tox pBAD-A & TGGGCTAGCGTGTGTATTGTCGTATCAGATG & Nhel \\
\hline Tox pBAD-B & TGCCGTCGACTTACCTCAATTTCAAACC & Sall \\
\hline HicBNde & GAGGTAAGCATATGCGCAAC & Ndel \\
\hline HicBXho & GGTATCTCGAGCACATITITG & Xhol \\
\hline RHHNde & AGGTTCATATGTCCGACGCC & Ndel \\
\hline ToxXbaT & CATCTAGAGAGCGGCGAC & Xbal \\
\hline ToxEcoT & GAATTCCTCAATTTCAAACCG & ECORI \\
\hline DopXbaT & CCTCTAGACAACTATATCGG & Xbal \\
\hline DopEcoT & AGGAATTCACATTITGCTGG & ECORI \\
\hline RHHXba & AATCTAGAATGTCCGACGCCGAGAACAGG & Xbal \\
\hline COGECO & TAGAATTCCAACCTCAAGGGAGGAGGG & ECORI \\
\hline RHHNde & CGGTGAAAACATATGCAAAAAGAG & Ndel \\
\hline HicBNH2Nde & GGCATATGCGCAACTATATCGGATTGATC & Ndel \\
\hline HicBNH2Xho & CCCTCGAGTTCGGCGAAGGCATCTATC & Xhol \\
\hline PET-Bgl & GGGGGGGAAGATCTAGAAATAATTTTGTTAAC & Bg/ll \\
\hline PET-EcoRI & CGGGAATTCAGCAAAAAACCCCTCAAG & ECORI \\
\hline EMSAD & GATCCGACGGTTCGAGACCATCC & none \\
\hline EMSAR & TCTTCGGGTGAGGAACGGTAACC & none \\
\hline
\end{tabular}

*the restriction sites are underlined

HicB COG1598 and RHH1 domains were cloned into pKT25, pKNT25, pUT18 and pUT18C using DopXbaT/ COGEco and RHHXba/DopEcoT primer pairs. BACTH analysis was performed as described for HicA-HicB interaction.

\section{Additional files}

Additional file 1: Figure S1. HicA/HicB interaction analysis using the BACTH system. For analysis of interactions between HicA and HicB, either one of the plasmids pKThicA, pNThicA, pKThicB, or pNThicB were transformed into the E. coli strain BTH101 reporter strains (a cya-deficient strain), followed by secondary transformation of any of the following: pUThicA, pUChicA, pUThiB, or pUThicB. Positive and negative controls were performed using pKT25-ZIP/pUT18-ZIP and pKNT25/pUT18C sets respectively. Positive interactions allow the reconstitution of adenylate cyclase activity and thus the expression of lacZ gene in the cya strain BTH101. $\beta$-galactosidase activity (Miller units) is the mean of three independent experiments. (PPTX $95 \mathrm{~kb}$ )

Additional file 2: Figure S2. Nucleotide sequence of hicA-hicB synthetic operon introduced into pEt22b(+). HicA residues are highlighted in yellow, strep tag in pink, HicB in green and his tag in red. (PPTX 99 kb)

Additional file 3: Figure S3. Alignments of HicA and HicB proteins with HicA and HicB homologues of E. coli, Y. pestis and S. pneumoniae using Clustal Omega [43]. Symbols indicate a conserved residue $(*)$, conservative mutation (:) and a semi-conservative mutation (.). The $\mathrm{H} 23$ residue critical for HicA activity [25] is shown in red. (PPTX $55 \mathrm{~kb}$ )

Additional file 4: Figure S4. Alignments of S. meliloti HicB with HicB of S. pneumoniae (A)and HicB3 of Y. pestis (B) using Phyre [44]. (PPTX 598 kb)

Additional file 5: Figure S5. S. meliloti HicB structure predicted by Phyre using HicB of $Y$. pestis and S. pneumoniae as template. (PPTX $80 \mathrm{~kb}$ )

Additional file 6: Figure S6. HicB domains interactions. For analysis of auto-interactions between HicB domains and their interaction with HicA, either one of the plasmids pKTCOG, pNTCOG, pKTRHH, pNTRHH, pKThicA or pNThicA were transformed into the E. coli strain BTH101 reporter strains (a cya-deficient strain), followed by secondary transformation of any of the following: PUTCOG, pUCCOG, pUTRHH, pUTCRHH or pUThicA. Positive and negative controls were performed using pKT25-ZIP/pUT18ZIP and pKNT25/pUT18 sets respectively (not shown). Positive interactions allow the reconstitution of adenylate cyclase activity and thus the expression of lacZ gene in the cya strain BTH101. $\beta$-galactosidase activity (Miller units) is the mean of three independent experiments. (PPTX $81 \mathrm{~kb}$ )

\section{Abbreviations}

BACTH: Bacterial adenylate cyclase two-hybrid; BSA: Bovine serum albumin; CFU: Colony forming units; COG: Clusters of orthologous groups; EMSA: Electrophoretic mobility shift assay; IPTG: Isopropyl $\beta$-D-1-thiogalactopyranoside; OD570nm: Optical density measured at $570 \mathrm{~nm}$; ON: Overnight: PBS: Phosphate-buffered saline; PCR: Polymerase chain reaction; RHH: Ribbon-helix-helix

\section{Acknowledgements}

We sincerely thank Sylvie Georgeault, Marie-Christine Savary and Lydie JoseTeixeira-Pinault from the IGDR for technical assistance. We also thank Prof. Reynald Gillet (University of Rennes1) head of IGDR, for his general support and for proofreading this manuscript.

\section{Funding}

This research was supported by the Region Bretagne for MT's grant ARED ("Allocations de Recherche Doctorale") Nº 9287/RIBOMOD "Ribosomes et Stress". The laboratory facilities were supported by the Centre National de la Recherche Scientifique (CNRS) and the University of Rennes1. The funding bodies had no role in designing the study, collection of strains, analysis and interpretation of data and in writing the manuscript.

\section{Availability of data and materials}

The datasets used and/or analyzed during the current study are available from the corresponding author on reasonable request. 


\section{Authors' contributions}

MT, AT, GE and CB conceived and designed the experiments. AT and CB constructed all the plasmids used in this publication. MT and CB performed the protein purification and BACTH assays. MT and GE was involved in ribosome, rRNA purifications and RNase assays. CB and GE wrote the paper. All authors read and approved the final manuscript.

\section{Ethics approval and consent to participate}

Not applicable.

\section{Consent for publication}

Not applicable.

\section{Competing interests}

The authors declare that they have no competing interest.

\section{Publisher's Note}

Springer Nature remains neutral with regard to jurisdictional claims in published maps and institutional affiliations.

\section{Received: 23 September 2018 Accepted: 26 December 2018}

\section{Published online: 10 January 2019}

\section{References}

1. Harms A, Brodersen DE, Mitarai N, Gerdes K. Toxins, targets, and triggers: an overview of toxin-antitoxin biology. Mol Cell. 2018;70(5):768-84.

2. Muthuramalingam $M$, White $J$, Bourne $C$. Toxin-antitoxin modules are pliable switches activated by multiple protease pathways. Toxins. 2016;8(7):214.

3. Wang X, Lord DM, Cheng H-Y, Osbourne DO, Hong SH, Sanchez-Torres V, Quiroga C, Zheng K, Herrmann T, Peti W, et al. A new type $V$ toxin-antitoxin system where mRNA for toxin GhoT is cleaved by antitoxin GhoS. Nat Chem Biol. 2012;8:855-61.

4. Aakre Christopher D, Phung Tuyen N, Huang D, Laub MT. A bacterial toxin inhibits DNA replication elongation through a direct interaction with the $\beta$ sliding clamp. Mol Cell. 2013;52(5):617-28.

5. Gerdes K, Christensen SK, Løbner-Olesen A. Prokaryotic toxin-antitoxin stress response loci. Nat Rev Microbiol. 2005:3:371-82.

6. Gerdes K. Toxin-antitoxin modules may regulate synthesis of macromolecules during nutritional stress. J Bacteriol. 2000;182(3):561-72.

7. Hõrak R, Tamman H. Desperate times call for desperate measures: benefits and costs of toxin-antitoxin systems. Curr Genet. 2017;63(1):69-74.

8. Page R, Peti W. Toxin-antitoxin systems in bacterial growth arrest and persistence. Nat Chem Biol. 2016;12:208-14.

9. Kwan BW, Lord DM, Peti W, Page R, Benedik MJ, Wood TK. The MasR/MasA toxin/antitoxin system protects Escherichia coli during bile acid stress. Environ Microbiol. 2015;17(9):3168-81.

10. Wang X, Lord DM, Hong SH, Peti W, Benedik MJ, Page R, Wood TK. Type II toxin/antitoxin MasR/MasA controls type V toxin/antitoxin GhoT/GhoS. Environ Microbiol. 2013;15(6):1734-44.

11. Hu Y, Benedik MJ, Wood TK. Antitoxin DinJ influences the general stress response through transcript stabilizer CspE. Environ Microbiol. 2012;14(3): 669-79.

12. Wang X, Kim Y, Hoon Hong S, Ma Q, Brown BL, Pu M, Tarone AM, Benedik MJ, Peti W, Page R, et al. Antitoxin MqsA helps mediate the bacterial general stress response. Nat Chem Biol. 2011;7(6):359-66.

13. De la Cruz MA, Zhao W, Farenc C, Gimenez G, Raoult D, Cambillau C, Gorvel J-P, Méresse S. A toxin-antitoxin module of Salmonella promotes virulence in mice. PLoS Pathog. 2013;9(12):e1003827.

14. Ren D, Walker AN, Daines DA. Toxin-antitoxin loci vapBC-1 and vapXD contribute to survival and virulence in nontypeable Haemophilus influenzae. BMC Microbiol. 2012;12:263.

15. Wen W, Liu B, Xue L, Zhu Z, Niu L, Sun B. Autoregulation and virulence control by the toxin-antitoxin system SavRS Instaphylococcus aureus. Infect Immun. 2018

16. Goormaghtigh F, Fraikin N, Putrinš M, Hallaert T, Hauryliuk V, Garcia-Pino A, Sjödin A, Kasvandik S, Udekwu K, Tenson T, et al. Reassessing the Role of Type II Toxin-Antitoxin Systems in Formation of <span class="namedcontent genus-species" id="named-content-1">Escherichia coli</span> Type II Persister Cells. mBio. 2018;9(3):e00640-18.

17. Holden DW, Errington J. Type II Toxin-Antitoxin Systems and Persister Cells. mBio. 2018;9(5):e01574-18.
18. Song S, Wood TK. Post-segregational Killing and Phage Inhibition Are Not Mediated by Cell Death Through Toxin/Antitoxin Systems. Frontiers in Microbiology. 2018;9(814).

19. Butt A, Higman Victoria A, Williams C, Crump Matthew P, Hemsley Claudia M, Harmer N, Titball RW. The HicA toxin from Burkholderia pseudomallei has a role in persister cell formation. Biochem J. 2014:459(2):333-44.

20. Button JE, Silhavy TJ, Ruiz N. A suppressor of cell death caused by the loss of $\sigma$ E downregulates Extracytoplasmic stress responses and outer membrane vesicle production in Escherichia coli. J Bacteriol. 2007;189(5): 1523-30.

21. Daimon Y, Narita S-I, Akiyama Y. Activation of toxin-antitoxin system toxins suppresses lethality caused by the loss of $\sigma E$ in Escherichia coli. J Bacteriol. 2015:197(14):2316-24.

22. Li G, Shen M, Lu S, Le S, Tan Y, Wang J, Zhao X, Shen W, Guo K, Yang Y, et al. Identification and characterization of the HicAB toxin-antitoxin system in the opportunistic pathogen Pseudomonas aeruginosa. Toxins. 2016;8(4):113.

23. Mhlanga-Mutangadura T, Morlin G, Smith AL, Eisenstark A, Golomb M. Evolution of the major pilus gene cluster of Haemophilus influenzae. J Bacteriol. 1998:180(17):4693-703.

24. Bibi-Triki S, Li de la Sierra-Gallay I, Lazar N, Leroy A, Van Tilbeurgh H, Sebbane F, Pradel E. Functional and structural analysis of HicA3-HicB3, a novel toxin-antitoxin system of Yersinia pestis. J Bacteriol. 2014;196(21): 3712-23.

25. Kim D-H, Kang S-M, Park SJ, Jin C, Yoon H-J, Lee B-J. Functional insights into the Streptococcus pneumoniae HicBA toxin-antitoxin system based on a structural study. Nucleic Acids Res. 2018;46(12):6371-86.

26. Makarova KS, Grishin NV, Koonin EV. The HicAB cassette, a putative novel, RNA-targeting toxin-antitoxin system in archaea and bacteria. Bioinformatics. 2006;22(21):2581-4.

27. Jorgensen MG, Pandey DP, Jaskolska M, Gerdes K. HicA of Escherichia coli defines a novel family of translation-independent mRNA interferases in bacteria and archaea. J Bacteriol. 2009:191(4):1191-9.

28. Sevin EW, Barloy-Hubler F. RASTA-Bacteria: a web-based tool for identifying toxin-antitoxin loci in prokaryotes. Genome Biol. 2007:8(8):R155.

29. Bodogai M, Ferenczi S, Bashtovyy D, Miclea P, Papp P, Dusha I. The ntrPR operon of Sinorhizobium meliloti is organized and functions as a toxinantitoxin module. Mol Plant-Microbe Interact. 2006;19(7):811-22.

30. Lipuma J, Cinege G, Bodogai M, Oláh B, Kiers A, Endre G, Dupont L, Dusha I. A vapBC-type toxin-antitoxin module of Sinorhizobium meliloti influences symbiotic efficiency and nodule senescence of Medicago sativa. Environ Microbiol. 2014;16(12):3714-29.

31. Milunovic B, GC dC, Morton RA, Finan TM. Cell growth inhibition upon deletion of four toxin-antitoxin loci from the Megaplasmids of Sinorhizobium meliloti. J Bacteriol. 2014;196(4):811-24.

32. Capela D, Barloy-Hubler F, Gouzy J, Bothe G, Ampe F, Batut J, Boistard P, Becker A, Boutry M, Cadieu E, et al. Analysis of the chromosome sequence of the legume symbiont Sinorhizobium meliloti strain 1021. Proc Natl Acad Sci U S A. 2001;98(17):9877-82.

33. Pradel E, Lemaitre N, Merchez M, Ricard I, Reboul A, Dewitte A, Sebbane F. New insights into how Yersinia pestis adapts to its mammalian host during bubonic plague. PLoS Pathog. 2014;10(3):e1004029.

34. Buts L, Lah J, Dao-Thi M-H, Wyns L, Loris R. Toxin-antitoxin modules as bacterial metabolic stress managers. Trends Biochem Sci. 2005;30(12):672-9.

35. Sallet E, Roux B, Sauviac L, Jardinaud MF, Carrere S, Faraut T, de CarvalhoNiebel F, Gouzy J, Gamas P. Capela D, et al. Next-generation annotation of prokaryotic genomes with EuGene-P: application to Sinorhizobium meliloti 2011. DNA Res. 2013;20(4):339-54.

36. Schlüter J-P, Reinkensmeier J, Barnett MJ, Lang C, Krol E, Giegerich R, Long SR, Becker A. Global mapping of transcription start sites and promoter motifs in the symbiotic a-proteobacterium Sinorhizobium meliloti 1021. BMC Genomics. 2013;14:156.

37. Karimova G, Pidoux J, Ullmann A, Ladant D. A bacterial two-hybrid system based on a reconstituted signal transduction pathway. Proc Nat Acad Sci U S A. 1998:95(10):5752-6.

38. Turnbull KJ, Gerdes K. HicA toxin of Escherichia coli derepresses hicAB transcription to selectively produce HicB antitoxin. Mol Microbiol. 2017; 104(5):781-92

39. Sambrook J, Fritsch EF, Maniatis T. Molecular cloning: a laboratory manual, 2nd ed., cold Spring Harbor edn. New York: Cold Spring Harbor; 1989.

40. Kovach ME, Elzer PH, Hill DS, Robertson GT, Farris MA, Roop RM, Peterson $\mathrm{KM}$. Four new derivatives of the broad-host-range cloning vector 
pBBR1MCS, carrying different antibiotic-resistance cassettes. Gene. 1995;166: 175-6.

41. Aranda PS, LaJoie DM, Jorcyk CL. Bleach gel: a simple agarose gel for analyzing RNA quality. Electrophoresis. 2012;33(2):366-9.

42. Miller JH. Experiments in molecular genetics. Cold Spring Harbor, NY: Cold Spring Harbor Laboratory; 1972

43. Sievers F, Higgins DG. Clustal omega for making accurate alignments of many protein sequences. Prot Sci. 2018;27(1):135-45.

44. Kelley LA, Mezulis S, Yates CM, Wass MN, Sternberg MJE. The Phyre2 web portal for protein modeling, prediction and analysis. Nat Protoc. 2015;10: $845-58$.

45. Jozefkowicz C, Brambilla S, Frare R, Stritzler M, Puente M, Piccinetti C, Soto $G$, Ayub N. Microevolution rather than large genome divergence determines the effectiveness of legume-rhizobia symbiotic interaction under field conditions. J Mol Evol. 2017:85(3-4):79-83.

46. Blattner FR, Plunkett G 3rd, Bloch CA, Perna NT, Burland V, Riley M, ColladoVides J, Glasner JD, Rode CK, Mayhew GF, et al. The complete genome sequence of Escherichia coli K-12. Science. 1997;277(5331):1453-62.

47. Studier WF, Moffat BA. Use of bacteriophage T7 RNA polymerase to direct selective high-level expression of cloned genes. J Mol Biol. 1986;189:113-30.

48. Battesti A, Bouveret E. The bacterial two-hybrid system based on adenylate cyclase reconstitution in Escherichia coli. Methods. 2012;58(4):325-34.

49. Guzman LM, Belin D, Carson MJ, Beckwith J. Tight regulation, modulation, and high-level expression by vectors containing the arabinose PBAD promoter. J Bacteriol. 1995;177(14):4121-30.

50. Gotfredsen M, Gerdes K. The Escherichia coli relBE genes belong to a new toxin-antitoxin gene family. Mol Microbiol. 1998;29(4):1065-76.

Ready to submit your research? Choose BMC and benefit from:

- fast, convenient online submission

- thorough peer review by experienced researchers in your field

- rapid publication on acceptance

- support for research data, including large and complex data types

- gold Open Access which fosters wider collaboration and increased citations

- maximum visibility for your research: over $100 \mathrm{M}$ website views per year

At $\mathrm{BMC}$, research is always in progress.

Learn more biomedcentral.com/submissions 